\title{
Arrhythmogenic effect of flecainide toxicity
}

\author{
Pierre-Yves Courand ${ }^{1}$, Franck Sibellas ${ }^{1}$, Sylvain Ranc ${ }^{1}$, Audrey Mullier ${ }^{2}$, \\ Gilbert Kirkorian ${ }^{1}$, Eric Bonnefoy ${ }^{1}$ \\ ${ }^{1}$ Intensive Care Unit of Cardiology, Louis Pradel Hospital, Hospices Civils de Lyon, France \\ ${ }^{2}$ Emergency Department, Jules Courmont Hospital, Hospices Civils de Lyon, France
}

\begin{abstract}
Flecainide is a class $1 C$ antiarrhythmic drug especially used for the management of supraventricular arrhythmia. In overdose cases, flecainide can induce life treating ventricular arrhythmias and cardiogenic shock. We report the case of a 72-year-old woman admitted to our intensive care unit for a regular monomorphic wide complex tachycardia (QRS duration $240 \mathrm{~ms}$, right bundle branch block and superior axis morphology) without apparent $P$ waves. Clinical examination showed slight left congestive heart failure signs without cardiogenic shock. An intravenous bolus of $10 \mathrm{mg}$ adenosine 5'-triphosphate (ATP) was ineffective to stop the tachycardia. The diagnosis of ventricular tachycardia induced by flecainide overdose was considered. $500 \mathrm{~mL}$ of intravenous $84 \%$ sodium bicarbonate was administrated. The patient's $Q R S$ narrowed immediately and 12-lead ECG showed sinus rhythm. Blood samples confirmed the flecainide overdose and the clinical status progressively improved. (Cardiol J 2013; 20, 2: 203-205)

Key words: flecainide, toxicity, overdose, ventricular arrhythmia, ATP
\end{abstract}

\section{Introduction}

Flecainide is a class $1 \mathrm{C}$ antiarrhythmic drug used especially for the management of supra-ventricular like atrial fibrillation $(\mathrm{AF})$ [1]. It causes ratedependent slowing of the rapid sodium channel slowing phase 0 of depolarisation and in high doses inhibits the slow calcium channel [2]. Flecainide also slows conduction in all cardiac fibers, increasing conduction times in the atria, ventricles, atrioventricular node and His-Purkinje system. Flecainide can also cause myocardial depression. In overdose cases, flecainide induced life treating ventricular arrhythmia and cardiogenic shock requiring sometimes extracorporeal membrane oxygenation $[3,4]$.

\section{Case report}

A 72-year-old woman was admitted to our intensive care unit for breathlessness in a context of a regular tachycardia with wide QRS complex. Her medical history included hypertension, paroxysmal $\mathrm{AF}$ and a significant psychiatric history. She was treated with flecainide (100 mg twice daily), irbesartan (300 mg daily) and haloperidol (1 mg twice daily). Of note, she had no beta-blocker therapy. Questioning did not find any voluntary drug intoxication. Her general practitioner performed three months ago a 12-lead ECG. It showed sinus rhythm with QTc interval of $400 \mathrm{~ms}$ and QRS duration of $100 \mathrm{~ms}$. Before her admission to our intensive care unit, she was given $150 \mathrm{mg}$ of intravenous amio-

Address for correspondence: Pierre-Yves Courand, MD, Intensive Care Unit of Cardiology, Louis Pradel Hospital, Hospices Civils de Lyon, 69317 Lyon, France, tel: 003347207 16 67, fax: 00334720716 74,

e-mail: pycourand@hotmail.com

Received: 24.04.2012 Accepted: 07.05.2012 


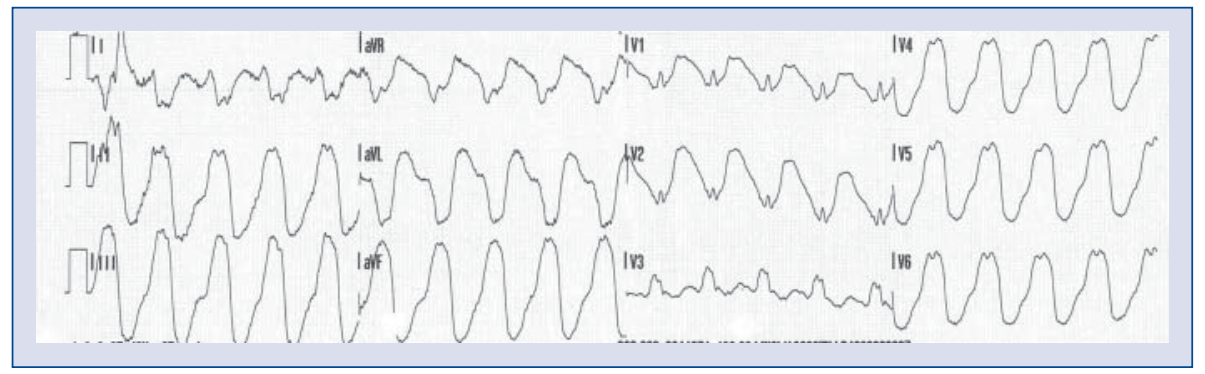

Figure 1. 12-lead ECG displays monomorphic wide complex (QRS duration $240 \mathrm{~ms}$, left bundle branch block inferior axis morphology) tachycardia at $150 \mathrm{bpm}$ without apparent $\mathrm{P}$ waves.

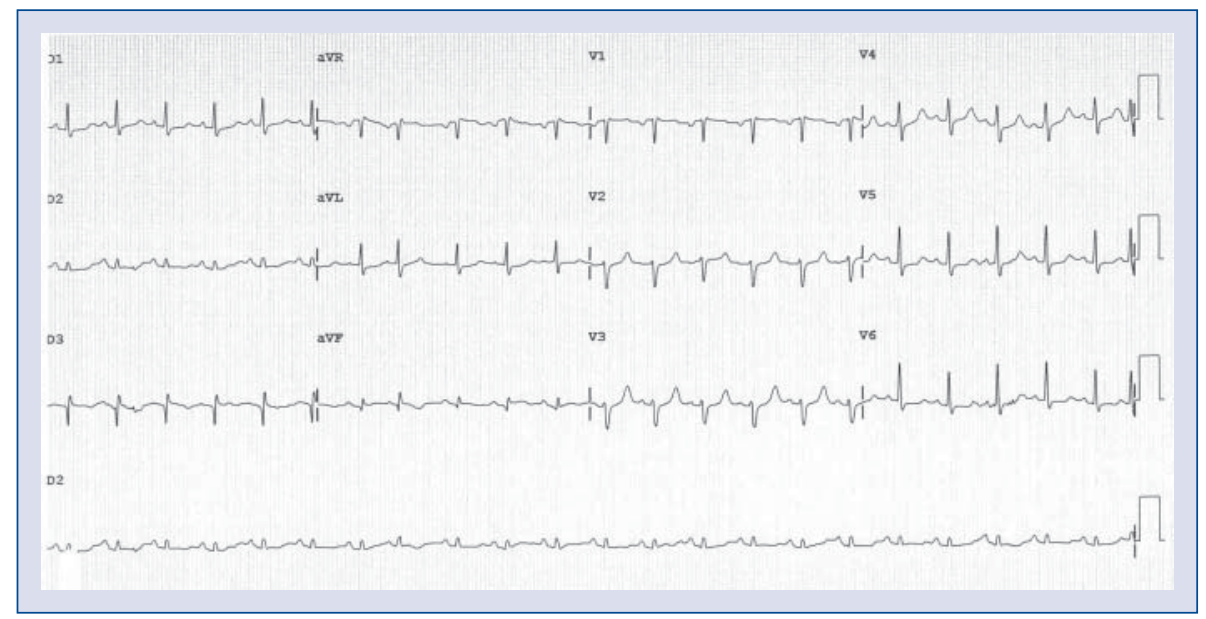

Figure 2. 12-lead ECG demonstrates a sinus rhythm after administration of $500 \mathrm{~mL}$ of intravenous $84 \%$ o sodium bicarbonate.

darone in the emergency room. Clinical examination showed slight left congestive heart failure signs without cardiogenic shock (blood pressure 100/60 mm $\mathrm{Hg}$, oxygen saturation 95\%). The 12-lead ECG showed a regular monomorphic wide complex tachycardia at $150 \mathrm{bpm}$ without apparent $\mathrm{P}$ wave (Fig. 1). QRS duration was around $240 \mathrm{~ms}$ with a left bundle branch block and inferior axis morphology. Transthoracic echocardiography showed a mild left ventricular hypertrophy with a moderate systolic dysfunction (left ventricular ejection fraction 35\%), a lack of atrial systole on trans-mitral flow and no valvular disease. An intravenous bolus of $10 \mathrm{mg}$ adenosine 5'-triphosphate (ATP) was used as a diagnostic tool to identify the mechanism of the tachycardia. This administration was ineffective to slow or stop the tachycardia. The diagnosis of ventricular tachycardia induced by flecainide overdose was considered and $500 \mathrm{~mL}$ of intravenous $84 \%$ sodium bicarbonate was administrated. The patient's QRS narrowed immediately following sodium bicarbonate infusion and 12-lead ECG showed sinus rhythm without evidence of accessory pathway (Fig. 2). Blood samples performed before sodium bicarbonate infusion demonstrated mild metabolic acidosis $(\mathrm{pH} 7.36$, normal $7.37-7.45$, arterial lactate $1.60 \mathrm{mmol} / \mathrm{L}$, normal $<1.20$ ), mild renal failure (estimated glomerular filtration rate with MDRD at $42 \mathrm{~mL} / \mathrm{min}$ ), BNP rise $(1019 \mathrm{ng} / \mathrm{L}$, normal value $<100)$ and a flecainide level at $2.13 \mathrm{mg} / \mathrm{L}$ (normal therapeutic range 0.2 $-1 \mathrm{mg} / \mathrm{L}$ ). Otherwise, troponin I level, blood cells count and serum electrolytes, especially kaliemia, were unremarkable. Clinical status progressively improved. On day 7, transthoracic echocardiography showed a left ventricular ejection fraction improvement up to $50 \%$ and the patient was discharged from hospital with a treatment associating amiodarone and irbesartan. One month later, the patient was admitted to our hospital for a check-up. The Holter ECG showed a permanent sinus rhythm without paroxysmal arrhythmias. The coronary angiogram ruled out any coronary artery stenosis. An electrophysiological study demonstrated no inducible ventricular or supraventricular arrhythmias. 


\section{Discussion}

Flecainide, a class $1 \mathrm{C}$ anti-arrhythmic agent, depresses the rate of depolarization of cardiac action potentials producing a membrane stabilizing action. It is a very effective anti-arrhythmic agent against supraventricular arrhythmias, nevertheless flecainide is contraindicated in patients with structural heart disease because it increased mortality [1]. The proarrhythmic effect of flecainide may be related to promoting a reentry in ventricular tissue. The phenomenon is due to a rate-dependent blockade of rapid sodium channels slowing phase 0 of depolarization and an inhibition of the slow calcium channel [2]. In cases of overdose, the mortality with class Ic agents has been reported to approach $22 \%$. Conduction disturbances began with widening of QRS complex which can rapidly progress to ventricular tachycardia, electromechanical dissociation and asystole. The markers of poor prognosis for overdose with membrane stabilizing action drugs are: ingested dose, heart rate $>100 \mathrm{bpm}, \mathrm{QRS}$ duration $>100 \mathrm{~ms}$ and QT elongation [5]. In the present report, predisposing factors of flecainide toxicity were left ventricular hypertrophy, metabolic acidosis, renal failure (flecainide is mainly excreted in urine), mild heart failure and a likely drug interaction with haloperidol. Of note, we did not found predisposing genetic factors in our patient. Although there are only few successfully treated cases reported [6, 7], hypertonic sodium bicarbonate is considered as a specific antidote to treat flecainide overdose. Hypertonic sodium bicarbonate increases extra cellular concentration of sodium displacing flecainide from its receptors sites. Because flecainide is a weak acid, alkalinization may also decrease the active-ionized fraction of flecainide necessary for sodium channels blockade [8]. In our case, sodium bicarbonate infusion significantly reduced QRS duration in few minutes and improved hemodynamic status. This case report tends to confirm the therapeutic efficiency of sodium bicarbonate in a case of moderate flecainide overdose.

ATP can be used as an effective diagnosis tool in identifying the mechanism of wide QRS tachycardia. ATP frequently terminated supraventricular tachycardia, but it had no effect on preexcited
AF or atrial flutter. ATP can also terminated ventricular tachycardia due to triggered-activity or automacity, while ventricular tachycardia related to reentry are insensitive to this drug [9]. Our report supports this data. During flecainide overdose, the mechanism of ventricular arrhythmias is also a ventricular reentry and thus ATP cannot terminate the tachycardia. Hence, ATP is normally considered an antiarrhythmic agent.

\section{Conclusions}

We illustrated with our report that ATP is not an effective antiarrythmic agent to terminate a ventricular arrhythmias due to flecainide toxicity. In this clinical setting, sodium bicarbonate should be preferred.

Conflict of interest: none declared

\section{References}

1. The Cardiac Arrhythmia Suppression Trial (CAST) Investigators. Preliminary report: Effect of encainide and flecainide on mortality in a randomized trial of arrhythmia suppression after myocardial infarction. N Engl J Med, 1989; 321: 406-412.

2. Krishnan SC, Antzelevitch C. Flecainide-induced arrhythmia in canine ventricular epicardium. Phase 2 reentry? Circulation, 1993; 87: 562-572.

3. Corkeron MA, van Heerden PV, Newman SM, Dusci L Extracorporeal circulatory support in near-fatal flecainide overdose. Anaesth Intensive Care, 1999; 27: 405-408.

4. Auzinger GM, Scheinkestel CD. Successful extracorporeal life support in a case of severe flecainide intoxication. Crit Care Med, 2001; 29: 887-890.

5. Koppel C, Oberdisse U, Heinemeyer G. Clinical course and outcome in class Ic antiarrhythmic overdose. J Toxicol Clin Toxicol, 1990; 28: 433-444.

6. Golman MJ, Mowry JB, Kirk MA. Sodium Bicarbonate to correct widened QRS in a case of flecainide overdose. J Emerg Med, 1997; 15:183-186.

7. Rognoni A, Bertolazzi M, Peron M et al. Electrocardiographic changes in a rare case of flecainide poisoning: A case report. Cases J, 2009; 3: 9137.

8. Bajaj AK, Woosley RL, Roden DM. Acute electrophysiologic effects of sodium bicarbonate administration in dogs treated with O-desmethyl encainide. Circulation, 1989; 80: 994-1002.

9. Kobayashi Y, Yazawa T, Adachi T et al. Ventricular arrythmias with left bundle branch block pattern and inferior axis-assessment of their mechanisms on the basis of the response to ATP, nicorandil and verapamil. Jpn Circ J, 2000; 64: 835-841. 